\title{
Detection of hydrogen peroxide with chemiluminescent micelles
}

\author{
Dongwon Lee' \\ Venkata R Erigala',3 \\ Madhuri Dasari' \\ Junhua $\mathrm{Yu}^{2}$ \\ Robert M Dickson ${ }^{2}$ \\ Niren Murthy' \\ 'The Wallace H. Coulter Department \\ of Biomedical Engineering; \\ ${ }^{2}$ Department of Chemistry and \\ Biochemistry, Georgia Institute of \\ Technology, Atlanta, GA, USA; ${ }^{3}$ The \\ Scripps Research Institute, La Jolla, \\ CA, USA
}

Correspondence: Niren Murthy

The Wallace H. Coulter Department

of Biomedical Engineering, Georgia

Institute of Technology, Atlanta,

GA 30332, USA

Tel + I 404385 I 329

Fax + I 4048944243

Email niren.murthy@bme.gatech.edu

\begin{abstract}
The overproduction of hydrogen peroxide is implicated in the progress of numerous life-threatening diseases and there is a great need for the development of contrast agents that can detect hydrogen peroxide in vivo. In this communication, we present a new contrast agent for hydrogen peroxide, termed peroxalate micelles, which detect hydrogen peroxide through chemiluminescence, and have the physical/chemical properties needed for in vivo imaging applications. The peroxalate micelles are composed of amphiphilic peroxalate based copolymers and the fluorescent dye rubrene, they have a 'stealth' polyethylene glycol (PEG) corona to evade macrophage phagocytosis, and a diameter of $33 \mathrm{~nm}$ to enhance extravasation into permeable tissues. The peroxalate micelles can detect nanomolar concentrations of hydrogen peroxide $(>50 \mathrm{nM})$ and thus have the sensitivity needed to detect physiological concentrations of hydrogen peroxide. We anticipate numerous applications of the peroxalate micelles for in vivo imaging of hydrogen peroxide, given their high sensitivity, small size, and biocompatible PEG corona.
\end{abstract}

Keywords: hydrogen peroixde, chemiluminescence, micelles, amphiphilic copolymer

\section{Introduction}

Hydrogen peroxide plays a central role in causing several life-threatening human disease, and there is currently great interest in developing contrast agents that can image hydrogen peroxide in vivo (Chang et al 2004; Miller et al 2005). The detection of hydrogen peroxide in vivo is challenging because of low reactivity and low concentration. The imaging of hydrogen peroxide in vivo was recently accomplished using a new family of nanoparticles, termed peroxalate nanoparticles (Lee et al 2007). The peroxalate nanoparticles are composed of polymeric peroxalate esters and fluorescent dyes, and detect hydrogen peroxide via a three component chemiluminescent reaction between a peroxalate ester, hydrogen peroxide and a fluorescent dye. Peroxalate nanoparticles had a mean size of $550 \mathrm{~nm}$ and exhibited excellent sensitivity and specificity to hydrogen peroxide. They were also capable of imaging hydrogen peroxide generated in the peritoneal cavity of mice during inflammation, after a direct intraperitoneal injection. However, desptite their great potential, these peroxalate nanoparticles are unsuitable for intravenous applications because their large size will prevent their extravasation into tissues, and their hydrophobic surface will promote clearance by the reticular endothelial system (RES) (Allen and Cullis 2004; Peer et al 2007).

In this report, we present a new hydrogen peroxide contrast agent, termed peroxalate micelles, which have the physicochemical properties needed for intravenous injections. The peroxalate micelles are composed of peroxalate containing amphiphilic polynorbornene copolymers and fluorescent dyes. Amphiphilic polynorbornene copolymers were synthesized via ring opening metathesis polymerization and self-assembled with fluorescent dyes, generating peroxalate micelles, which have a mean size of $33 \mathrm{~nm}$ and a 'stealth' polyethylene glycol (PEG) coating to enhance their circulation 
time (Torchilin 2002; Allen and Cullis 2004). Similar to the peroxalate nanoparticles, the peroxalate micelles image hydrogen peroxide by undergoing a three component chemiluminescent reaction, catalyzed by hydrogen peroxide, which results in the emission of photons and, therefore, the detection of hydrogen peroxide. The small size and nanomolar sensitivity $(>50 \mathrm{nM})$ of the peroxlate micelles make them potentially useful for in vivo imaging of hydrogen peroxide.

\section{Materials and methods}

\section{Materials}

5-Norbornene-2-methanol, oxalic chloride, 5-norbornene2-carboxylic acid, monomethoxy PEG $(\mathrm{Mn}=2,000)$ and Grubbs catalyst (1st generation) were purchased from Sigma-Aldrich (MO. USA). All reagents were used without further purification.

\section{Synthesis of norbornenylphenyl oxalate, (I)}

5-Norbornene-2-methanol $(8 \mathrm{mmol})$ and phenol $(8 \mathrm{mmol})$ were dissolved in $20 \mathrm{~mL}$ of dry dichloromethane under nitrogen, to which triethylamine $(15 \mathrm{mmol})$ was added, at $0{ }^{\circ} \mathrm{C}$. Oxalic chloride $(8 \mathrm{mmol})$ was added to the mixture dropwise at $0{ }^{\circ} \mathrm{C}$ and the reaction was kept at room temperature overnight. The reaction mixture was extracted with dichloromethane, dried on sodium sulfate, filtered, and concentrated to get the crude compound $\mathbf{1 . 1}$ was purified using silica gel chromatography (hexane/ethyl acetate $=90: 10)($ yield 40\%). ${ }^{1} \mathrm{H}-\mathrm{NMR}\left(\mathrm{CDCl}_{3}, 400 \mathrm{MHz}\right.$ spectrometer from Varian)

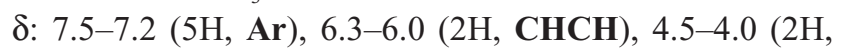
CH2OCO), 3.0-2.6 (2H, CHCH2CH), 1.9 (1H, CHCH2O), 1.6-1.2 (2H, CHCH2CH). EIMass $(m / z)=272\left(\mathrm{M}^{+}\right)$.

\section{Synthesis of norbornene-ended poly(ethyleneglycol), (2)}

Monomethoxy PEG (2.5 $\mathrm{mmol})$ and 5-norbornene2 -carboxylic acid $(2.9 \mathrm{mmol})$ were dissolved in dry dichloromethane $(60 \mathrm{~mL})$. Dicyclohexyl carbodiimide (2.9 mmol) and a catalytic amount of 4-dimethylaminopyridine were added to the mixture and stirred at room temperature overnight. The resulting solid was removed by filtration and the filtrate was concentrated and precipitated in dichloromethane/hexane $(1: 2)$ to obtain the compound 2 (yield 80\%). ${ }^{1} \mathrm{H}-\mathrm{NMR}\left(\mathrm{CDCl}_{3}, 400 \mathrm{MHz}\right.$ spectrometer from Varian) $\delta$ : 6.3-6.0 (m, 2H, СНСН), 3.8-3.3 (m, 4H, OCH2СH2O), 3.0-2.6 (m, 2H, CHCH2CH), 1.9 (m, 1H, CHCH2O), 1.6-1.2 (m, 2H, CHCH2CH).

\section{Synthesis of poly(norbornenyl peroxalate)-poly(norbornenyl ethyleneglycol), (3)}

The compound $1\left(2.2 \times 10^{-4} \mathrm{~mol}\right)$ and Grubbs catalyst $\left(4.4 \times 10^{-6} \mathrm{~mol}\right)$ were dissolved in dry dichloromethane $(2 \mathrm{~mL})$ under nitrogen and the mixture was kept at room temperature for $30 \mathrm{~min}$. The compound $2\left(4.5 \times 10^{-5} \mathrm{~mol}\right.$ in $0.5 \mathrm{~mL}$ of dichloromethane) was added to the reaction mixture. The polymerization was kept at room temperature overnight and quenched with ethyl vinyl ether. The resulting polymer, $\mathbf{3}$, was isolated by precipitating in cold ether and concentrated under vacuum (yield 85\%). Gel permeation chromatography was performed in tetrahydrofuran (flow rate $=1 \mathrm{~mL} / \mathrm{min}$ ) on a Shimazhu chromatograph SCL-10A (Tokyo, Japan). The molecular weight of polymer 3 was determined to be $38,000 \mathrm{~g} / \mathrm{mol}$ (polydispersity = 1.5) using polystyrene standards.

\section{Chemiluminescent quantum yields of the compound I}

A dry THF solution $(1.5 \mathrm{~mL})$ containing the compound $1\left(2 \times 10^{-6} \mathrm{M}\right)$ and rubrene $\left(4 \times 10^{-5} \mathrm{M}\right)$ was placed in a $1 \mathrm{~cm} \times 1 \mathrm{~cm}$ quartz cuvette. Chemiluminescence profiles of signal versus time were acquired immediately after the addition of $0.5 \mathrm{~mL}$ of hydrogen peroxide $\left(3 \times 10^{-1} \mathrm{M}\right)$ for 2 min. The total chemiluminescent quantum yields $\left(\Phi_{\mathrm{CL}}\right)$ were calculated according to the following equation

$$
\Phi_{\mathrm{CL}}=G N\left(R_{\text {photo }} / M\right)
$$

where $N$ is the average number of counts, $R_{\text {photo }}$ the correction factor for maximum fluorescence emission of rubrene, $M$ the moles of the compound $\mathbf{1}$, and $G$ the correction factor for the spectrometer (Motoyoshiya et al 2002).

\section{Micelle preparation and characterization}

Peroxalate micelles were prepared via solvent displacement method. In brief, rubrene (1 mg) and copolymer 3 (20 mg) were dissolved in $1 \mathrm{~mL}$ of acetone, to which $9 \mathrm{~mL}$ of phosphate buffer ( $\mathrm{pH} 7.4,0.1 \mathrm{M}$ ) was added. The mixture was vortexed for $10 \mathrm{sec}$ and acetone was evaporated using a rotary evaporator, generating rubrene-encapsulated peroxalate micelles. The size of micelles at the concentration of $1 \mathrm{mg} / \mathrm{mL}$ was measured by dynamic light scattering (Particle Size Analyzer 90 Plus, Brookhaven Instrument Corporation, NY, US). Various amounts of hydrogen peroxide solution (10 $\mu \mathrm{M}$ in phosphate buffer $\mathrm{pH} 7.4,0.1 \mathrm{M}$ ) were added to 
each micelle solution. The micelles $(1 \mathrm{mg} / \mathrm{mL})$ were incubated with a range of hydrogen peroxide ( 0 to $1 \mu \mathrm{M})$ and their chemiluminescence was measured with a luminometer (Femtomaster FB12, Zylux Corporation, TN, US) with a $10 \mathrm{~s}$ acquisition time. The chemiluminescence emission spectrum of micelles was acquired in the presence of hydrogen peroxide $(10 \mu \mathrm{M})$ using a fluorometer (Photon Technology International, NJ, US).

\section{Results and discussion Molecular design of peroxalate micelles}

Peroxalate chemiluminescence is a three component reaction between hydrogen peroxide, a peroxalate ester, and a fluorescent dye and has great potential for hydrogen peroxide imaging because of its high sensitivity and excellent specificity (Stevani et al 2000; Arnous et al 2002; Matsumoto 2004). However, the application of peroxalate chemiluminescence for in vivo imaging of hydrogen peroxide is challenging because it requires sequestering fluorescent dyes and peroxalate esters within close proximity in vivo. Peroxalate micelles are designed to sequester peroxalate esters and fluorescent dyes in their core, enabling the three component peroxalate chemiluminescent reaction to occur in response to hydrogen peroxide. The peroxalate micelles (Figure 1) are composed of the amphiphilic polynorbornene copolymer 3 and rubrene, which was chosen as a fluorescent dye because of its high fluorescence quantum efficiency (Stevani et al 2000).

The copolymer 3 is composed of a hydrophobic polynorbornene backbone, which has peroxalate esters and PEG chains grafted to it. This amphilic graft copolymer is designed to self-assemble, in aqueous solutions, with hydrophobic fluorescent dyes, such as rubrene, generating micelles that have peroxalate esters and fluorescent dyes in their hydrophobic core and a PEG corona. The peroxalate micelles detect hydrogen peroxide through a two-step chemiluminescent process, as shown in Figure 1. First, hydrogen peroxide diffuses into the core of the micelles and reacts with peroxalate esters, immediately generating a high energy dioxetanedione intermediate, which then chemically excites encapsulated fluorescent dyes, through a CIEEL (chemically initiated electron exchange luminescence) mechanism, leading to photon emission and the detection of hydrogen peroxide

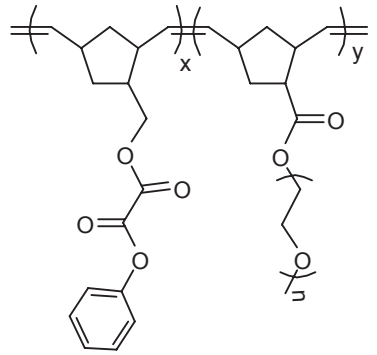

Amphiphilic polynorbornene copolymer (3)

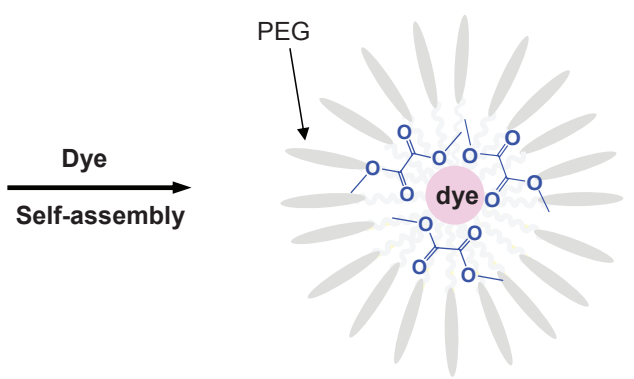

Peroxalate micelles encapsulating fluorescent dyes (4)

$\mathrm{H}_{2} \mathrm{O}_{2}$
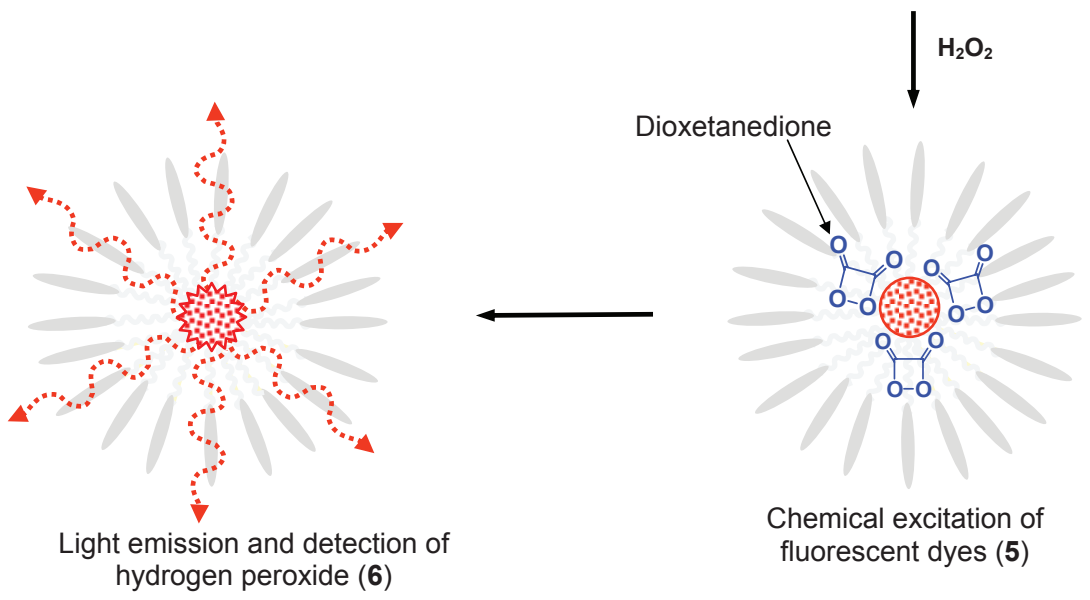

Chemical excitation of fluorescent dyes (5)

Figure I Peroxalate micelles: a new contrast agent for the detection of hydrogen peroxide. The copolymer (3) forms the scaffold of the peroxalate micelles and contains peroxalate esters and hydrophilic PEG chains. The peroxalate micelles (4) are formed by self-assembly of 3 with fluorescent dyes in aqueous solutions. Hydrogen peroxide diffuses into the micelles and reacts with its peroxalate esters, generating a high energy dioxetanedione intermediate which then chemically excites encapsulated dyes (5), leading to photon emission and the detection of hydrogen peroxide (6). 
(Matsumoto 2004; Koike et al 2006). One of unique features of the peroxalate micelles is their specificity to hydrogen peroxide. This is because peroxalae chemiluminescence requires the generation of a dioxetanedione intermediate, which can be formed only by hydrogen peroxide, and not by other reactive oxygen species (Lee et al 2007).

\section{Synthesis of amphiphilic polynorbornene copolymers}

A major challenge in developing contrast agents based on peroxalate chemiluminescence is a lack of synthetic techniques for generating polymeric peroxalate esters. The only current methodology for the synthesis of linear polymeric peroxalate esters is through the reaction of oxalyl chloride with diols. However, this polymerization method is not suitable for the development of amphiphilic block copolymers that have well-defined structures. In this study, ring opening metathesis polymerization (ROMP) was employed as a new route for the synthesis of amphiphilic peroxalate copolymers. A ROMP-based synthetic strategy was chosen because ROMP is an efficient and powerful method to synthesize amphiphilic copolymers which have a well-defined structure (Stubenrauch et al 2006; Feng et al 2002). In addition, peroxalate esters which are unstable in the presence of nucleophiles and water can tolerate ROMP reaction conditions (Scheme 1).

The copolymer 3 was synthesized via ROMP of the monomers $\mathbf{1}$ and $\mathbf{2}$ and had a molecular weight of 38,000 (PDI = 1.5). The ROMP monomer, bicyclo[2.2.1]hept5-en-2-ylmethyl phenyl oxalate (1) was designed as the peroxalate ester component of the micelles and was prepared in $40 \%$ yield from the reaction of norbornene-methanol, phenol, and oxalyl chloride. $\mathbf{1}$ is a mixed peroxalate ester, containing an aromatic and an aliphatic peroxalate ester. A mixed peroxalate ester was chosen as the hydrogen peroxide sensing moiety of $\mathbf{1}$ because of its straightforward synthesis and potential higher stability in aqueous solutions. The hydrophilic component of the peroxalate micelles was generated from the macromonomer $\alpha$-norbornenylpoly(eth yleneglycol), 2, which was synthesized from a reaction of 5-norbornene-2-carboxylic acid and monomethoxy PEG (Heroquez et al 1996; Heroquez et al 1997). Norbornenylp oly(ethyleneglycol) macromonomers have been used previously to synthesize amphiphilic branched copolymers that self-assemble into micelles (Table 1).

The vast majority of peroxalate esters used for peroxalate chemiluminescence are based on aromatic peroxalate esters such as diphenyl oxalate and trichlorophenyl oxalate. These phenolic based peroxalate esters react exceptionally well with hydrogen peroxide because of their low pKas (Rauhut 1969; Hadd et al 1999). The peroxalate ester used in this study is a mixed peroxalate ester (1) containing both an aromatic group and an aliphatic group, which has not been commonly investigated for peroxalate chemiluminescence. A potential limitation of using $\mathbf{1}$ for peroxalate chemiluminescence is its lower reactivity to hydrogen peroxide in comparison to aromatic peroxalate esters such as diphenyl oxalate, due to the higher $\mathrm{pKa}$ of aliphatic alcohols. In order to evaluate the sensitivity of $\mathbf{1}$ to hydrogen peroxide, we investigated the chemiluminescence quantum yield of $\mathbf{1}$ and compared it to diphenyl oxalate. The quantum efficiency of $\mathbf{1}$ and diphenyl oxalate was obtained at a concentration of $2 \times 10^{-6} \mathrm{M}$

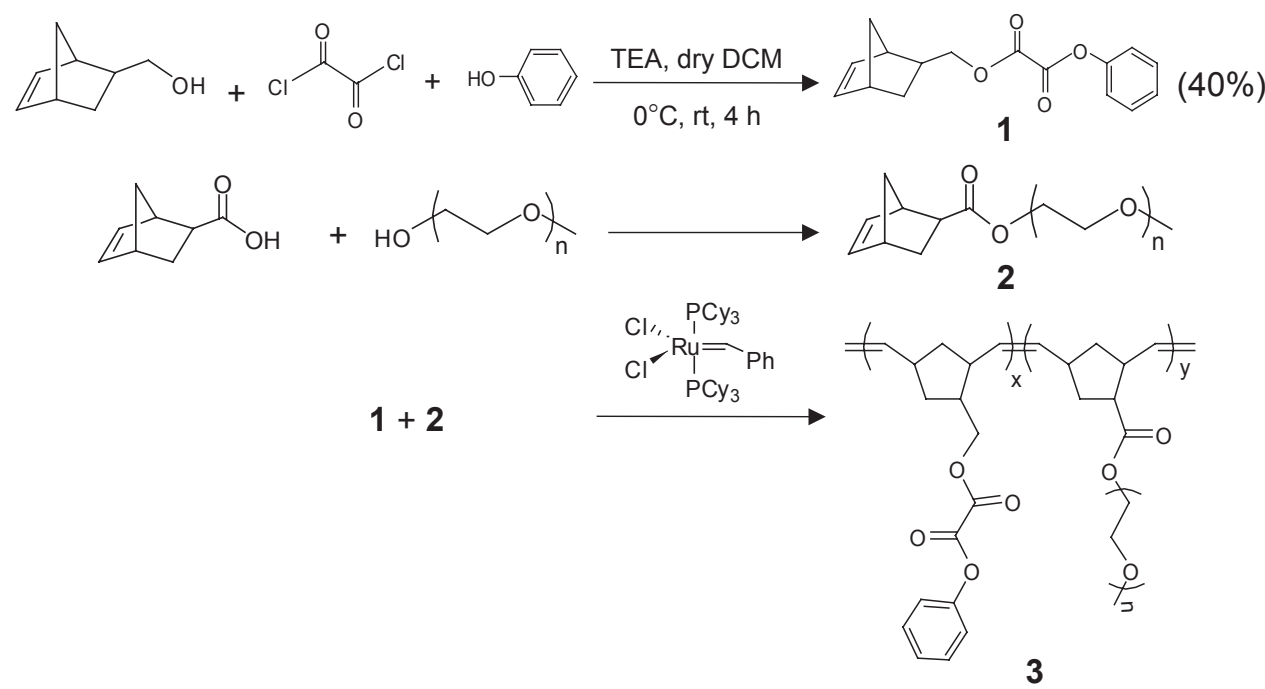

Scheme I Synthesis of amphiphilic polynorbornene copolymer (3) by ROMP. 
Table I Chemiluminescence quantum yields of peroxalate esters (I vs diphenyl oxalate)

\section{Substrates} Quantum efficiency

$\left(\Phi_{\mathrm{CL}} \times 10^{-7}\right)$

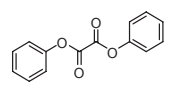

$2.34 \pm 0.18$

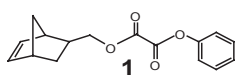

$1.98 \pm 0.13$

The chemiluminescence quantum efficiency of I and diphenyl oxalate was obtained by the photocounting method, using luminol chemiluminescence as a standard. I has a quantum efficiency comparable to that of diphenyl oxalate. Indicated values are means $( \pm S D)$ of two experiments.

with a large excess of rubrene. Table 1 demonstrates, under these experimental conditions, the quantum efficiency of $\mathbf{1}$ is comparable to that of diphenyl oxalate, suggesting that $\mathbf{1}$ has potential for hydrogen peroxide detection.

\section{Characterization of peroxalate micelles}

The peroxalate micelles were formulated from the copolymer 3 via a solvent displacement method. The solvent displacement method was chosen for micelle formation because it has been extensively used to generate micelles in the range of $20 \sim 50 \mathrm{~nm}$ in diameter. Micelles of this size are particularly useful in biological applications because of their long circulation time in the blood and great potential for extravasation into tissues (Gao et al 2002; Nasongkla et al 2006). The copolymer $3(20 \mathrm{mg})$ and fluorescent dye rubrene $(1 \mathrm{mg})$ were dissolved in acetone, followed by mixing with a large volume of water, generating micelles that had a mean size of $33 \mathrm{~nm}$ in diameter, as determined by dynamic light scattering (Figure 2a). The chemiluminescent emission spectrum of the micelles was obtained in the presence of hydrogen peroxide $(10 \mu \mathrm{M})$. Figure $2 \mathrm{~b}$ demonstrates that hydrogen peroxide initiates peroxalate chemiluminescence within the micelles, leading to photon emission at $560 \mathrm{~nm}$, which is similar to the fluorescence emission wavelength of rubrene.

\section{Sensitivity of the peroxalate micelles to hydrogen peroxide}

The ability of the peroxalate micelles to detect hydrogen peroxide was investigated by measuring their chemiluminescence intensity in the presence of hydrogen peroxide at various concentrations in the range of $0-1 \mu \mathrm{M}$. The intracellular concentration of hydrogen peroxide appears to be in the range of $1 \mathrm{nM}$ to $700 \mathrm{nM}$ (Stone 2004; Miller et al 2005). The Figure 3 demonstrates that the peroxalate micelles have excellent sensitivity to hydrogen peroxide and exhibit a linear correlation between chemiluminescence intensity and hydrogen peroxide concentration. The peroxalate micelles were capable of detecting hydrogen peroxide at concentrations as low as $50 \mathrm{nM}(18603 \mathrm{RLU} / \mathrm{s}$ at $50 \mathrm{nM}$ vs $252 \mathrm{RLU} / \mathrm{s}$ at $0 \mathrm{nM}$ ), suggesting great potential for the detection of hydrogen peroxide at physiological concentrations. The half-life of peroxalate chemiluminescence was approximately $1.5 \mathrm{~min}$ in the presence of hydrogen peroxide. Interestingly, the peroxalate micelles have a sensitivity for hydrogen peroxide that is 5 -fold greater than the larger peroxalate nanoparticles $(50 \mathrm{nM}$ vs $250 \mathrm{nM}$ ) (Lee et al 2007). We speculate that the enhanced sensitivity of the peroxalate micelles is partially due to their smaller size and higher surface area, which should enhance the diffusion of hydrogen peroxide into the micelles.

\section{Conclusion}

In this report, we present a new contrast agent for hydrogen peroxide, termed peroxalate micelles, which are composed of amphiphilic peroxalate copolymers and rubrene. The peroxalate micelles have peroxalate esters and rubrene in their hydrophobic core and a stealth PEG corona, and detect hydrogen peroxide by performing a three-component a

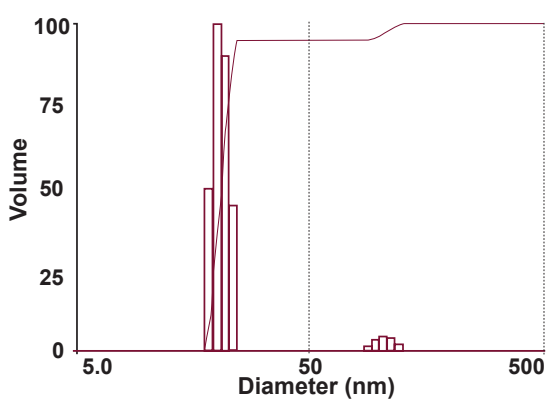

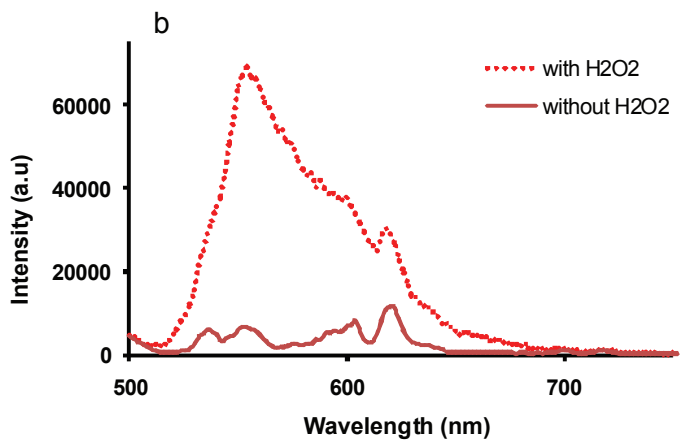

Figure 2 Dynamic light scattering (a) and chemiluminescence emission spectra of the peroxalate micelles (b). The emission wavelength was recorded in the absence or presence of hydrogen peroxide $(10 \mu \mathrm{M})$. 


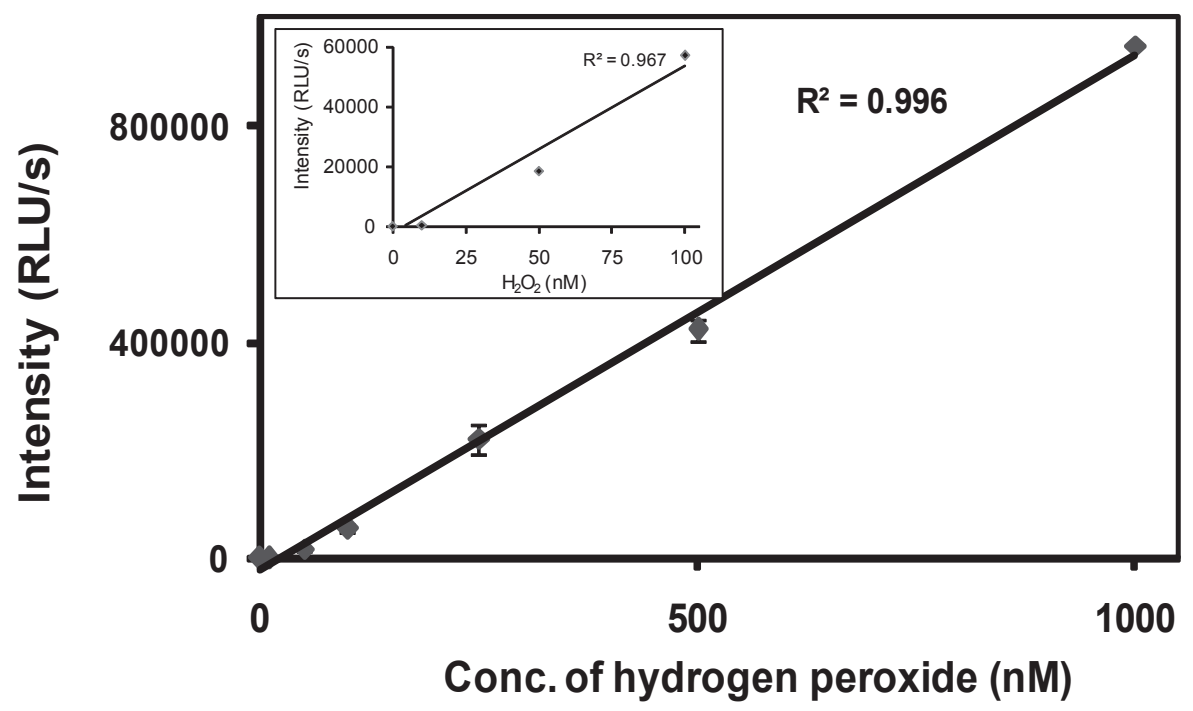

Figure 3 Sensitivity of peroxalate micelles to hydrogen peroxide. Data were obtained from I $\mathrm{mL}$ of micelles (I mg/mL) in response to increasing concentrations of hydrogen peroxide. RLU, relative light intensity. Mean $\pm S D, n=3$. The inset shows the chemiluminescence response to hydrogen peroxide in the range of 0 to $I 00 \mathrm{nM}$.

chemiluminescence reaction. The peroxalate micelles have a mean diameter of $33 \mathrm{~nm}$ and were capable of detecting hydrogen peroxide at concentrations as low as $50 \mathrm{nM}$. Peroxalate micelles have great potential for imaging of hydrogen peroxide in vivo, given their physicochemical properties and nanomolar sensitivity.

\section{Acknowledgments}

This work was supported by the Georgia Tech/Emory Center for the Engineering of Living Tissues (funded by NSF-EEC-9731643) (NM), NSF-BES-0546962 CAREER AWARD (NM), NIH UO1 HL80711-01 (NM), NIH R21 EB006418 (NM), and J\&J/GT Health Care Innovation Seed Grant Proposal (NM).

\section{References}

Allen TM, Cullis PR. 2004. Drug delivery systems: Entering the mainstream. Science, 303:1818-22.

Arnous A, Petrakis C, Makris DP, et al. 2002. A peroxyoxalate chemiluminescence-based assay for the evaluation of hydrogen peroxide scavenging activity employing 9,10-diphenylanthracene as the fluorophore. J Pharmacol Toxicol Methods, 48:171-7.

Chang MCY, Pralle A, Isacoff EY, et al. 2004. A selective, cell-permeable optical probe for hydrogen peroxide in living cells. $\mathrm{J} \mathrm{Am} \mathrm{Chem} \mathrm{Soc,}$ 126:15392-3.

Feng XS, Yan LF, Wen J, et al. 2002. Synthesis and self-assembly study of two-armed polymers containing crown ether core. Polymer, 43:3131-7.

Gao ZG, Lukyanov AN, Singhal A, et al. 2002. Diacyllipid-polymer micelles as nanocarriers for poorly soluble anticancer drugs. Nano Lett, 2:979-82.

Hadd AG, Lehmpuhl DW, Kuck LR, et al. 1999. Chemiluminescence demonstration illustrating principles of ester hydrolysis reactions. $J$ Chem Educ, 76:1237-40.

Rauhut MM. 1969. Chemiluminescence from concerted peroxide decomposition reactions. Acc Chem Res, 2:80-7.
Heroguez V, Breunig S, Gnanou Y, et al. 1996. Synthesis of alphanorbornenylpoly (ethylene oxide) macromonomers and their ringopening metathesis polymerization. Macromolecules, 29:4459-64.

Heroguez V, Gnanou Y, Fontanille M. 1997. Novel amphiphilic architectures by ring-opening metathesis polymerization of macromonomers. Macromolecules, 30:4791-8.

Koike R, Kato Y, Motoyoshiya J, et al. 2006. Unprecedented chemiluminescence behaviour during peroxyoxalate chemiluminescence of oxalates with fluorescent or electron-donating aryloxy groups. Luminescence, 21:164-73.

Lee D, Khaja S, Velasquez-Castano JC, et al. 2007. In vivo imaging of hydrogen peroxide with chemiluminescent nanoparticles. Nat Mater, 6:765-9.

Miller EW, Albers AE, Pralle A, et al. 2005. Boronate-based fluorescent probes for imaging cellular hydrogen peroxide. $J \mathrm{Am}$ Chem Soc, 127:16652-9.

Matsumoto M. 2004. Advanced chemistry of dioxetane-based chemiluminescent substrates originating from bioluminescence. Journal of Photochemistry and Photobiology C-Photochemistry Reviews, 5:27-53.

Motoyoshiya J, Hotta M, Nishii Y, et al. 2002. Peroxyoxalate chemiluminescence of $\mathrm{N}, \mathrm{N}$ '-bistosyl-1H, 4H-quinoxaline-2, 3-dione and related compounds. Dependence on electronic nature of fluorophores. J Org Chem, 67:7314-8.

Nasongkla N, Bey E, Ren J, et al. 2006. Multifunctional polymeric micelles as cancer-targeted, MRI-ultrasensitive drug delivery systems. Nano Lett, 6:2427-30.

Peer D, Karp JM, Hong S, et al. 2007. Nanocarriers as an emerging platform for cancer therapy. Nat Nanotechnol, 2:751-60.

Stevani CV, Silva SM, Baader WJ. 2000. Studies on the mechanism of the excitation step in peroxyoxalate chemiluminescence. Eur J Org Chem, 24:4037-46.

Stone JR. 2004. An assessment of proposed mechanisms for sensing hydrogen peroxide in mammalian systems. Arch Biochem Biophys, 422:119-24.

Stubenrauch K, Moitzi C, Fritz, G, et al. 2006. Precise tuning of micelle, core, and shell size by the composition of amphiphilic block copolymers derived from ROMP investigated by DLS and SAXS. Macromolecules, 39:5865-74.

Torchilin VP. 2002. PEG-based micelles as carriers of contrast agents for different imaging modalities. Adv Drug Deliv Rev, 54:235-52. 